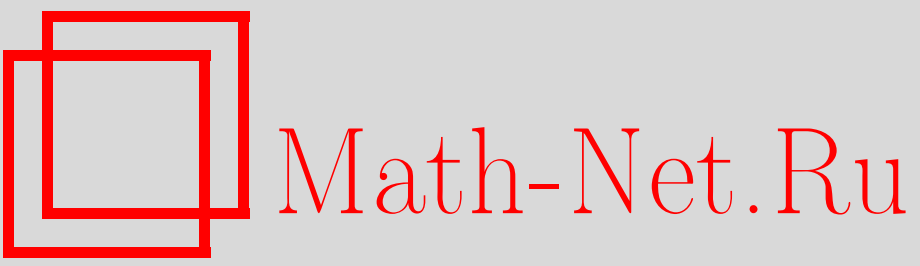

И. М. Буркин, О. И. Кузнецова, Об одном подходе к генерированию экстремально мультистабильных хаотических систем, Итоги науки и техн. Сер. Соврем. мат. и ее прил. Темат. обз., 2019, том 168, 15-25

DOI: https://doi.org/10.36535/0233-6723-2019-168-15-25

Использование Общероссийского математического портала Math-Net.Ru подразумевает, что вы прочитали и согласны с пользовательским соглашением

http: //www. mathnet.ru/rus/agreement

Параметры загрузки:

IP: 3.80 .181 .102

26 апреля 2023 г., 10:32:06 


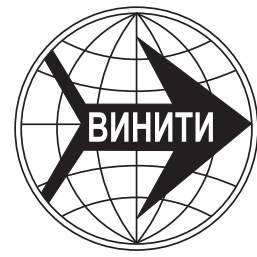

ИТОГИ НАУКИ И ТЕХНИКИ.

Современная математика и ее приложения.

Тематические обзоры.

Том 168 (2019). С. 15-25

DOI: 10.36535/0233-6723-2019-168-15-25

УДК 517.925

\title{
ОБ ОДНОМ ПОДХОДЕ К ГЕНЕРИРОВАНИЮ ЭКСТРЕМАЛЬНО МУЛЬТИСТАБИЛЬНЫХ ХАОТИЧЕСКИХ СИСТЕМ
}

\author{
(c) 2019 г. И. М. БУРКИН, О. И. КУЗНЕЦОВА
}

\begin{abstract}
АннотАция. В работе предлагаются новые подходы к построению экстремально мультистабильных систем, содержащих одномерные, двумерные и трехмерные решетки идентичных скрытых хаотических аттракторов, имеющих одинаковые показатели Ляпунова. В частности, показано, что для многомерных моделей систем автоматического управления в форме Лурье всегда возможен переход от системы в интерактивном режиме к системе каскадного типа, что существенно облегчает процедуру построения многомерных решеток идентичных хаотических аттракторов.
\end{abstract}

Ключевые слова: динамическая система, хаос, экстремальная мультистабильность, смещение фазового потока, показатели Ляпунова, размерность Каплана-Йорке.

\section{AN APPROACH TO GENERATING EXTREMELY MULTISTABLE CHAOTIC SYSTEMS}

\author{
(c) 2019 I. M. BURKIN, O. I. KUZNETSOVA
}

\begin{abstract}
In this paper, we propose new approaches to the construction of extremely multistable systems containing one-, two-, and three-dimensional lattices of identical hidden chaotic attractors possessing the same Lyapunov exponents. In particular, we prove that for multidimensional models of automatic control systems in the Lurie form, it is always possible to pass from interactive system to a cascade system; this substantially simplifies the procedure for constructing multidimensional lattices of identical chaotic attractors.
\end{abstract}

Keywords and phrases: dynamical system, chaos, extreme multistability, displacement of phase flow, Lyapunov exponent, Kaplan-Yorke dimension.

AMS Subject Classification: 34C28, 34D08, 37N35

1. Введение. В реальном физическом мире мультистабильность является достаточно распространенным явлением. Ее можно наблюдать, например, в динамических моделях газового лазера (см. [3]), биологических систем (см. [13]), волоконных лазеров (см. [9]), нейронных сетей (см. [27]), полупроводниковых суперрешеток (см. [26]). Мультистабильность динамической системы может создать угрозу в практических инженерных приложениях, поскольку поведение системы не может быть однозначно гарантировано. Такая система может демонстрировать решения с принципиально различным поведением в зависимости от выбора их начальных условий. C другой стороны, мультистабильность системы может являться ее неоспоримым преимуществом в том случае, когда она используется, например, для скрытия информации в системах коммуникаций (см. [7]) и аудиосхемах шифрования для повышения производительности защищенной связи (см. [22]), поскольку в данном случае специальный выбор начальных условий может играть роль 
«секретного ключа». По этой причине в последнее время изучению мультистабильных систем посвящено большое число работ (см. $[1,4,11,12,16,17,23,25])$.

Недавно в серии работ [18-20] был рассмотрен класс динамических систем, названных системами, допускающими смещение сдвигом по переменным (offset boostable dynamical systems). $\mathrm{K}$ таким системам относятся, в частности, системы гunepmoлчка (hyperjerk systems). Система гипертолчка $n$-го порядка может быть записана в виде эквивалентной системы каскадного типа в $\mathbb{R}^{n}$. В системе каскадного типа легко осуществить такое преобразование сдвига ее координат (смещение), которое меняет положение аттракторов системы в ее фазовом пространстве, не меняя в то же время динамику некоторых переменных. Последнее обстоятельство, в свою очередь, доставляет возможность трансформации системы каскадного типа в динамическую систему, обладающую бесконечной решеткой сосуществующих идентичных аттракторов, путем введения в нее периодических функций. Такие системы называют экстремально мультистабильными или бесконечно мультистабильными (см. [18]). В [18,20] построены бесконечно мультистабильные трехмерные системы, обладающие двумерной решеткой самовозбуждающихся аттракторов. В [19] на базе четырехмерной системы, допускающей смещение сдвигом, также построена система с трехмерной решеткой самовозбуждающихся гиперхаотических аттракторов, а также система без состояний равновесия, обладающая четырехмерной решеткой скрытых аттракторов. Напомним, что аттрактор системы называют самовозбуждающимся (self-excited attractor), если любая окрестность некоторого состояния равновесия системы пересекается с областью притяжения аттрактора; в противном случае аттрактор называется скрытым (hidden attractor) (см. [15]).

Для систем в $\mathbb{R}^{n}$, не являющимися системами каскадного типа, упомянутая процедура генерирования атрракторов-клонов также может быть реализована. Однако в этом случае, как отмечено в [19], процедура введения периодических функций намного сложнее, поскольку она может существенно повлиять на динамику системы и привести к разрушению ее аттракторов. В настоящее время известно огромное количество работ, в которых исследуются системы, обладающие самовозбуждающимися или скрытыми аттракторами (см., например, обзоры $[8,10])$. Поэтому естественным образом возникает вопрос: в каком случае система, обладающая хаотическим самовозбуждающимся или скрытым аттрактором, может быть приведена при помощи неособого линейного преобразования к системе каскадного типа, на базе которой путем введения периодическихх функиий может быть построена экстемально мультистабильная система?

В разделе 2 настоящей работы классическая система Чуа, содержащая скрытый хаотический аттрактор, использована для генерирования системы, содержащей одномерную решетку идентичных скрытых аттракторов. В разделах 2.1 и 2.2 рассмотрена система гипертолчка четвертого порядка, содержащая самовозбуждающийся хаотический аттрактор. Путем введения в эту систему тригонометрических функций строятся системы, содержащие двумерные и трехмерные решетки идентичных скрытых аттракторов. В разделе 3 показано, что для систем автоматического регулирования в форме Лурье с одной нелинейностью всегда существует такое неособое линейное преобразование координат, которое приводит эту систему к системе каскадного типа. Учитывая эту особенность систем в форме Лурье, удается, в частности, построить систему с двумерной решеткой идентичных скрытых хаотических аттракторов, используя классическую систему Чуа. Для всех построенных в работе экстремально мультистабильных систем вычисляются показатели Ляпунова и размерность Каплана-Йорке содержащихся в них аттракторов-клонов.

2. Приемы генерирования экстремально мультистабильных систем. Следуя работе [20] будем говорить, что динамическая система $\dot{X}=F(X), X=\left(x_{1}, x_{2}, \ldots, x_{i}, \ldots, x_{n}\right)$ является системой допускающей сдвиг по переменной $x_{i}$ (variable-boostable system), если замена переменной $x_{i}$ на $x_{i}+c$ при подходящем выборе значения постоянной $c$ не меняет динамику остальных переменных в исходном фазовом пространстве. Хорошо известным примером систем, допускающих сдвиг по переменной, являются так называемые фазовые системы автоматического управления вида,

$$
\dot{X}=A X+b \varphi(\sigma), \quad \sigma=c^{T} X
$$




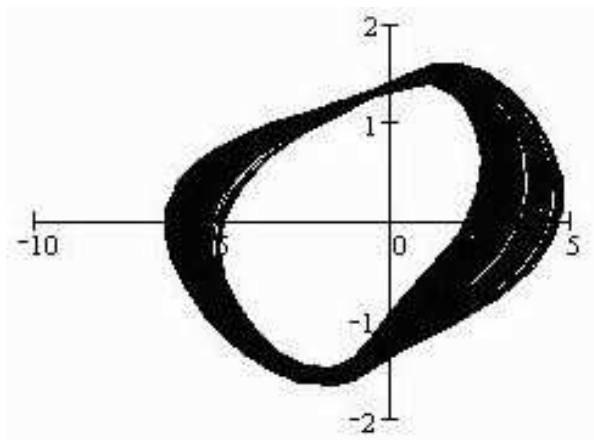

Рис. 1. Скрытый аттрактор классической системы Чуа

где $A-(n \times n)$-матрица, имеющая нулевое собственное значение, $b$ и $c-n$-векторы, $\varphi(\sigma)-\Delta$ периодическая функция. Как показано в [14], система (1) всегда может быть записана в виде

$$
\dot{Y}=B Y+q \varphi(\sigma), \quad \dot{\sigma}=r^{T} Y+\rho \varphi(\sigma),
$$

где $B-(n-1) \times(n-1)$-матрица, $q$ и $r-(n-1)$-векторы, $\rho$ - число. Поскольку в цилиндрическом фазовом пространстве $\left\{\left(y_{1}, y_{2}, \ldots, y_{n-1}, \sigma \bmod \Delta\right)\right\}$ динамический поток системы $(2)$ не меняется при замене $\sigma$ на $\sigma+k \Delta, k \in \mathbb{Z}$, то в накрывающем пространстве $\left\{\left(y_{1}, y_{2}, \ldots, y_{n-1}, \sigma\right)\right\}$ такая замена влечет за собой сдвиг фазового потока на $k \Delta$ в направлении оси $\sigma$, не меняя при этом динамику остальных переменных. Если фазовая система имеет аттрактор, целиком расположенный в «полосе» $\left\{y_{1}, y_{2}, \ldots, y_{n-1}, \sigma: y_{i} \in(-\infty ; \infty), \sigma \in\left(\sigma_{0} ; \sigma_{0}+\Delta\right)\right\}$, то она имеет бесконечную одномерную решетку идентичных аттракторов-клонов.

Приведенные соображения подсказывают естественный путь генерирования экстремально мультистабильных систем, обладающих одномерной решеткой идентичных аттракторов. Предположим, что известна система вида (1) с особой матрицей $A$, имеющая самовозбуждающийся или скрытый аттрактор, целиком расположенный в полосе $\left\{X: \Delta_{0} \leqslant c^{T} X \leqslant \Delta_{0}+\Delta\right\}$. Заменив в $(1)$ функцию $\varphi(\sigma)$ на $\Delta$-периодическую функцию $\psi(\sigma)$, совпадающую с $\varphi(\sigma)$ на $[0 ; \Delta]$, получим систему с одномерной решеткой идентичных аттракторов.

Приведем реализацию описанного алгоритма построения бесконечно мультистабильной системы на примере клонирования скрытого аттрактора классической системы Чуа. В безразмерных координатах такая система может быть записана в виде

$$
\dot{x}=\alpha(y-x)-\alpha g(x), \quad \dot{y}=x-y+z, \quad \dot{z}=-\beta y-\gamma z,
$$

(см. [6]), где функция $g(x)$ характеризует нелинейный элемент («диод Чуа»). Классической системой Чуа называют систему (3) с нелинейностью вида

$$
g(x)=m_{1} x+0,5\left(m_{0}-m_{1}\right)(|x+1|-|x-1|) .
$$

В соотношениях (3)-(4) $\alpha, \beta, \gamma, m_{0}, m_{1}$ - параметры классической системы Чуа.

Следуя работе [6], выберем параметры системы следующим образом: $\alpha=8,4562, \beta=12,0732$, $\gamma=0,0052, m_{0}=-0,1768, m_{1}=-1,1468$. Запишем систему (3)-(4) в виде (1) с

$$
A=\left(\begin{array}{ccc}
-\alpha\left(m_{1}+1\right) & \alpha & 0 \\
1 & -1 & 0 \\
0 & -\beta & -\gamma
\end{array}\right), \quad b=\left(\begin{array}{c}
-\alpha \\
0 \\
0
\end{array}\right), \quad c=\left(\begin{array}{l}
1 \\
0 \\
0
\end{array}\right), \quad \varphi(\sigma)=g(\sigma)-m_{1} \sigma .
$$

Такая система была подробно исследована в [5], где было показано, что она обладает скрытым хаотическим аттрактором, который может быть обнаружен путем численного интегрирования с начальным условием $X_{0}=(3,414309,1,41477,-3,666077)$. Этот аттрактор представлен на рис. 1. При этом $\left|c^{T} X\right| \leqslant 6,35$.

Заменим в системе $(1)-(5)$ функцию $\varphi(\sigma)$ на периодическую функцию $\psi(\sigma)$ с периодом $\Delta=$ 13,1766 , совпадающую с $\varphi(\sigma)$ на периоде $\Delta$. Новая система, как и ожидалось, обладает бесконечным числом скрытых аттракторов, представленных на рис. 2. 


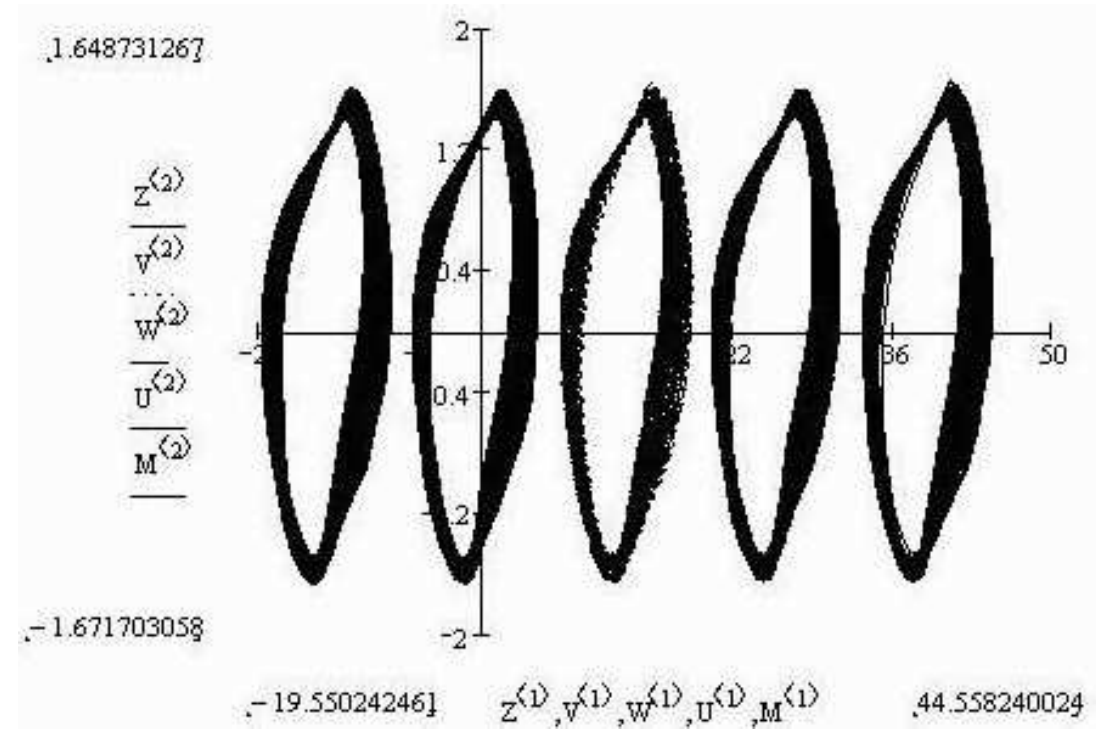

Рис. 2. Одномерная решетка скрытых аттракторов системы Чуа с периодической нелинейностью

Точки, принадлежащие скрытым аттракторам одномерной решетки, представленной на рис. 2 , отыскивались следующим образом. Сначала было найдено такое значение $k$, что матрица $\widetilde{A}=$ $A+k b c^{T}$ имеет нулевое собственное значение. Пусть $s$-соответствующий этому собственному значению собственный вектор матрицы $\widetilde{A}$. Положим $d=\Delta s\left(c^{T} s\right)^{-1}$. Тогда точки $X_{j}=X_{0}+j d$, $j=0, \pm 1, \pm 2, \ldots$, принадлежат аттракторам одномерной решетки.

Ясно, что продемонстрированный поход не может быть использован для генерирования на основе системы (3) экстремально мультистабильной системы, обладающей решеткой скрытых аттракторов размерности $>1$. В $[19,20]$ рассмотрен другой класс систем, позволяющих производить смещение фазового потока путем сдвига по переменным (offset boostable systems). Речь идет о системах каскадного типа, полученных из систем толчка и гипертолчка. Система гипертолчка описывается уравнением

$$
\frac{d^{n} x}{d t^{n}}=f\left(\frac{d^{n-1} x}{d t^{n-1}}, \ldots, \frac{d x}{d t}, x\right), \quad n \geqslant 4
$$

При $n=3$ это система толчка. В случае $n=4$ система (6) может быть трансформирована в систему каскадного типа, допускающую, например, трехмерный сдвиг по переменным, путем введения трех новых переменных $y, z, u$ следующим образом:

$$
\dot{x}=y, \quad \dot{y}=z, \quad \dot{z}=u, \quad \dot{u}=f(\dot{x}, \dot{y}, \dot{z}, x) .
$$

Сдвиг по переменным $y, z, u$ в системе (7) может быть осуществлен путем введения дополнительных констант в ее первые три уравнения. В самом деле, производя замену переменных $x \rightarrow \widetilde{x}$, $y \rightarrow \widetilde{y}+m, z \rightarrow \widetilde{z}+n, u \rightarrow \widetilde{u}+p$, приходим к системе

$$
\begin{aligned}
& \dot{\tilde{x}}=\widetilde{y}+m, \\
& \dot{\widetilde{y}}=\widetilde{z}+n, \\
& \dot{\widetilde{z}}=\widetilde{u}+p, \\
& \dot{u}=f(\dot{\tilde{x}}, \dot{\tilde{y}}, \dot{\widetilde{z}}, \widetilde{x}) .
\end{aligned}
$$

Легко видеть, что выполненное преобразование системы осуществляет смещение фазового потока системы (7) по переменным $y, z, u$, оставляя неизменной динамику переменной $x=\widetilde{x}$, поскольку динамика этой переменной подчиняется тому же самому уравнению (6). Очевидно, сдвиг можно осуществить отдельно по любой из указанных переменных или по любой паре из них. Если в системе (7) переменные $y, z, u$ заменить на периодические функции $G(y), R(z), F(u)$ и при 


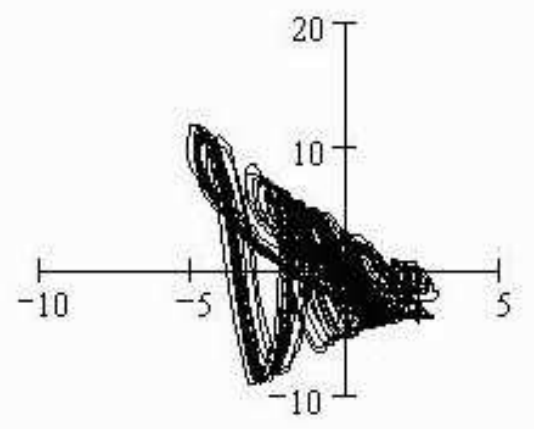

Рис. 3. Самовозбуждающийся аттрактор системы (9)

этом полученная система имеет аттрактор, то, как нетрудно убедиться (см. [19]), что она имеет бесконечную трехмерную решетку идентичных аттракторов. В $[18,20]$ для каскадных трехмерных систем вида (7) путем замены переменных $y$ и $z$ на тригонометрические функции построены бесконечно мультистабильные трехмерные системы, обладающие двумерной решеткой самовозбуждающихся аттракторов.

В качестве примера мы рассмотрим систему гипертолчка четвертого порядка:

$$
\dddot{x}=-\dddot{x}-3 \dot{x}-x-e^{\ddot{x}}-2 \ddot{x} e^{-\ddot{x}^{2}}+1,
$$

для которой соответствующая система каскадного типа (7) имеет вид

$$
\begin{aligned}
& \dot{x}=y, \\
& \dot{y}=z, \\
& \dot{z}=u, \\
& \dot{u}=-x-3 y-u-e^{z}-2 z e^{-z^{2}}+1 .
\end{aligned}
$$

Эта система имеет единственное неустойчивое состояние равновесия $(0,0,0,0)$ типа седло-фокус с собственными значениями матрицы линеаризации $(-0,515 \pm 2,365 i, 0,015 \pm 0,413 i)$ и самовозбуждающийся из окрестности состояния равновесия аттрактор, представленный на рисю 3 . Ниже будет продемонстрировано, каким образом путем введения в систему (9) периодических функций могут быть построены динамические системы, обладающие двумерной или трехмерной решеткой скрытых хаотических аттракторов-клонов.

2.1. Построение двумерной решетки скрытых аттракторов. Заменим в системе (9) переменные $y, u$ на периодические функции $F(y)=a \sin (b y), G(u)=c \sin (m u)$ и подберем значения параметров $a, b, c, m$ так, чтобы новая система имела устойчивое в малом состояние равновесия $(0,0,0,0)$ и хаотический аттрактор, который, очевидно, будет скрытым. Характеристический полином матрицы Якоби новой системы в точке $(0,0,0,0)$ имеет вид $r(p)=p^{4}+c m p^{3}+3 c m p^{2}+$ $3 a b c m p+a b c m$. Полином $r(p)$ будет полиномом Гурвица, например, при $a=2,1, b=1 / 2, c=5$, $m=1 / 3$. При этом система

$$
\begin{aligned}
& \dot{x}=2,1 \sin \frac{y}{2}, \\
& \dot{y}=z, \\
& \dot{z}=5 \sin \frac{u}{3}, \\
& \dot{u}=-x-6,3 \sin \frac{y}{2}-5 \sin \frac{u}{3}-e^{z}-2 z e^{-z^{2}}+1
\end{aligned}
$$

имеет скрытый хаотический аттрактор с ляпуновскими показателями $\Lambda_{1}=0,018, \Lambda_{2}=0,000$, $\Lambda_{3}=-0,055, \Lambda_{4}=-1,354$ и размерностью Каплана-Йорке $D_{K Y}=2+\left(\Lambda_{1}+\Lambda_{2}\right)\left|\Lambda_{3}\right|^{-1}=2,296$. Проекция этого аттрактора на плоскость $(y, u)$ представлена на рис. 4. 


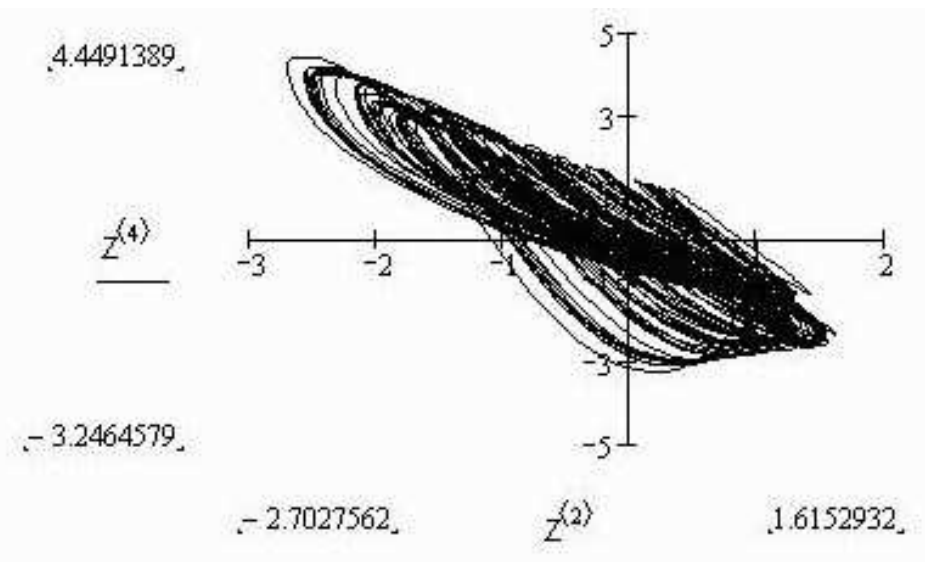

Рис. 4. Проекция скрытого аттрактора системы (10) на плоскость $(y, u)$

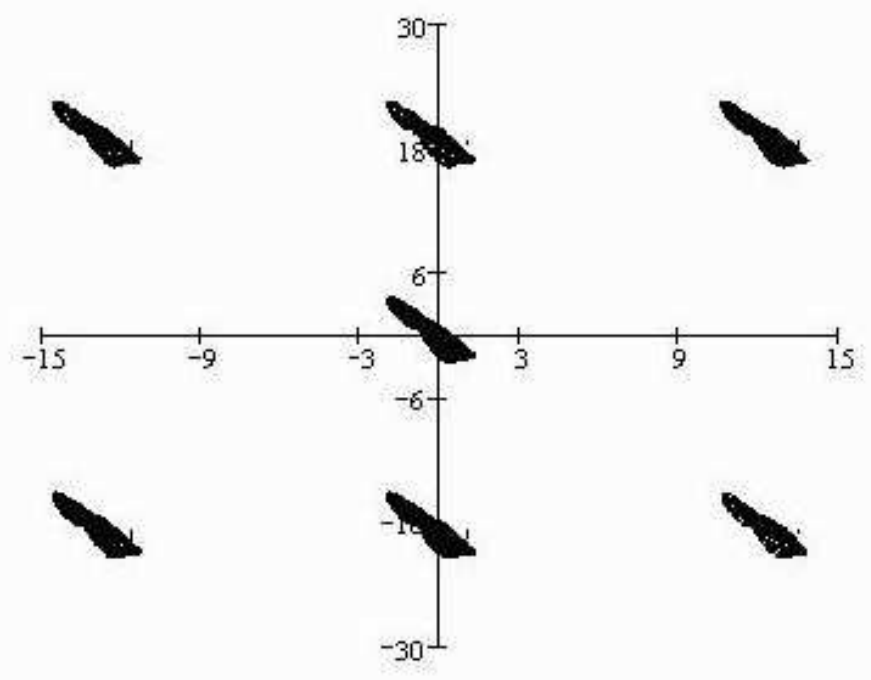

Рис. 5. Двумерная решетка скрытых аттракторов системы (10)

Замена в системе (9) переменных $y, u$ на периодические функции обеспечивает существование в фазовом пространстве системы (10) бесконечной двумерной решетки скрытых хаотических аттракторов-клонов с идентичными характеристиками - ляпуновскими показателями и размерностью Каплана-Йорке. Шесть аттракторов-клонов, полученные сдвигом аттрактора, представленного на рис. 4 на периоды $\pm 4 \pi$ и $\pm 6 \pi$ по осям $O y$ и $O u$ соответственно, представлены на рис. 5.

2.2. Построение трехмерной решетки скрытых аттракторов. Так как система (9) допускает смещение фазового потока по переменным $y, z$ и $u$, то можно попытаться заменить эти переменные на функции, периодические по переменным $y, z$ и $u$ так, чтобы новая система имела бесконечную трехмерную решетку идентичных скрытых аттракторов.

Путем целенаправленного компьютерного поиска реализовать описанный сценарий удалось в виде системы

$$
\begin{aligned}
& \dot{x}=\operatorname{tg} y, \\
& \dot{y}=\operatorname{tg} z, \\
& \dot{z}=1,1 \operatorname{tg} u, \\
& \dot{u}=-x-3 \operatorname{tg} y-1,15 \operatorname{tg} u-e^{\operatorname{tg} z}-2 \operatorname{tg} z e^{-\operatorname{tg}^{2} z}+1 .
\end{aligned}
$$




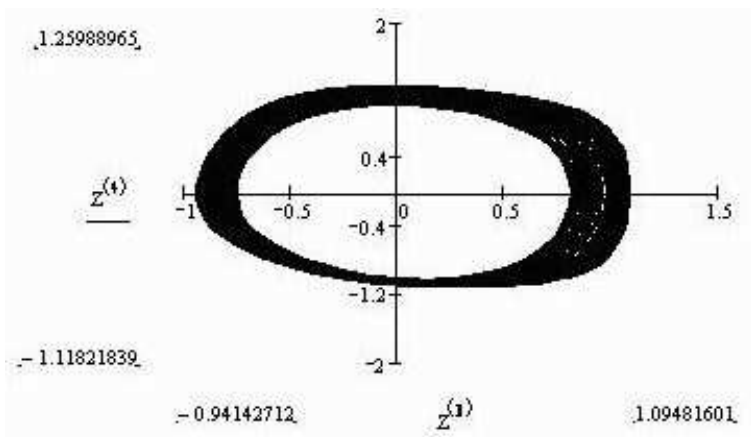

Рис. 6. Проекция аттрактора на плоскость $(z, u)$

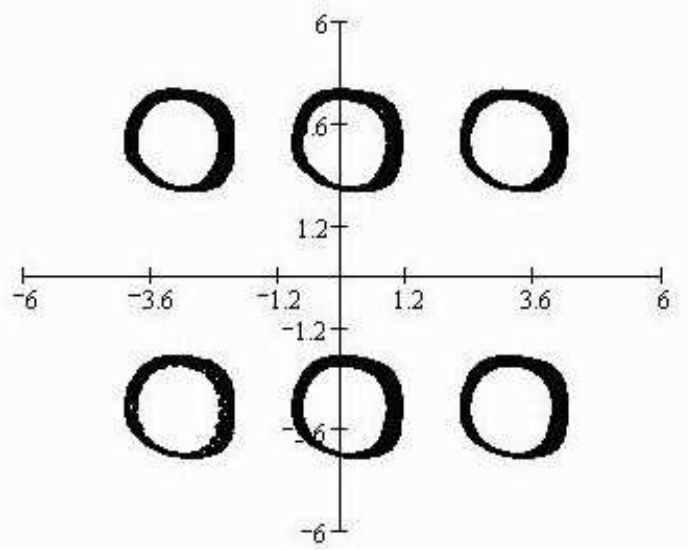

Рис. 8. Проекция аттракторов-клонов на плоскость $(z, u)$

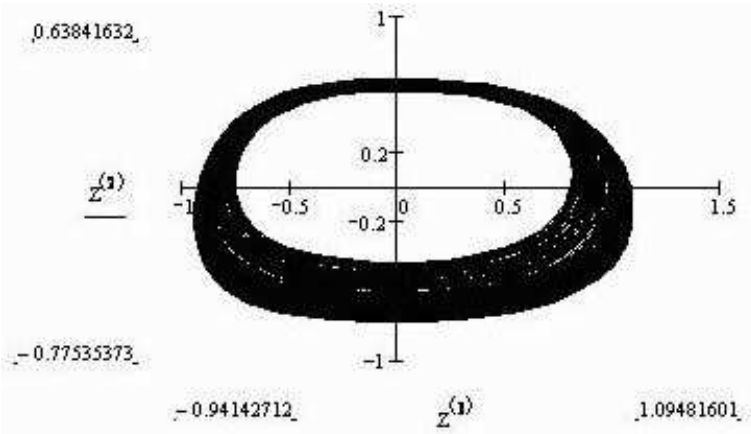

Рис. 7. Проекция аттрактора на плоскость $(y, z)$

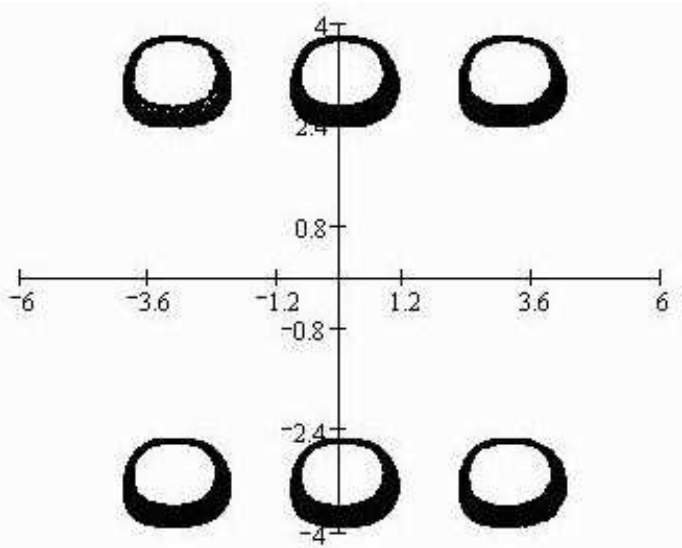

Рис. 9. Проекция аттракторов-клонов на плоскость $(y, z)$

Матрица линеаризации системы (11) в состоянии равновесия $(0,0,0,0)$ имеет собственные значения $(-0,011 \pm 1,746 i,-0,564 \pm 0,242 i)$, поэтому все ее состояния равновесия асимптотически устойчивы в малом. Решение с начальным условием $(-0,1,0,6,0,-0,1)$ находится в области притяжения скрытого хаотического аттрактора с ляпуновскими показателями $\Lambda_{1}=0,164, \Lambda_{2}=-0,139$, $\Lambda_{3}=-0,229, \Lambda_{4}=-2,944$ и размерностью Каплана-Йорке $D_{K Y}=2,107$. Проекции этого аттрактора на плоскости $(z, u)$ и $(y, z)$ представлены на рис. 6 и 7.

На рис. 8 и 9 представлены аттракторы-клоны, полученные сдвигом указанного аттрактора на периоды $\pm \pi$ по осям $O z, O u$ и $O y, O z$.

\section{3. Генерирование эстремально мультистабильных систем с использованием систем} регулирования в форме Лурье. В предыдущем разделе экстремально мультистабильные системы, содержащие многомерные решетки скрытых хаотических аттракторов, были построены с использованием смещаемых по переменным систем каскадного типа, которые, в свою очередь, были получены из систем гипертолчка (6). Для систем, не являющихся системами каскадного типа (систем в интерактивном режиме [20]), процедура введения периодических функций, позволяющая построить систему, содержащую многомерную решетку аттракторов, оказывается много сложнее. Такая процедура может существенно менять динамику системы и приводить к разрушению ее аттракторов. Необходимое преобразование системы удается осуществить, как правило, только с использованием целенаправленного компьютерного поиска (см. [19,20]).

В настоящем разделе мы покажем, что многомерная модель системы автоматического регулирования (1) в форме Лурье всегда может быть приведена при помощи неособого линейного 
преобразования к системе каскадного типа. Последнее обстоятельство позволяет, в частности, использовать многие известные результаты, связанные с существованием самовозбуждающихся или скрытых аттракторов у систем вида $(1)$ (см. $[8,10])$, для генерирования экстремально мультистабильных систем.

Пусть $I$ - единичная $(n \times n)$-матрица. Для системы $(1)$ определим дробно-рациональную функцию комплексного аргумента $p$ следующим образом: $\chi(p)=c^{T}(A-p I)^{-1} b$. Пусть $\chi(p)=$ $m(p)[n(p)]^{-1}$, причем многочлен в знаменателе дроби $\chi(p)$ имеет степень $n$ и несократим с ее числителем. В этом случае говорят, что передаточная функция $\chi(p)$ невырождена (см. [2]). Хорошо известно следующее утверждение (см. [2]): две системы вида (1) с одной и той же передаточной функиией эквивалентны с точностъю до неособого линейного преобразования их координат. Если

$$
\chi(p)=\frac{c_{0}+c_{1} p+\cdots+c_{n-1} p^{n-1}}{a_{0}+a_{1} p+\cdots+a_{n-1} p^{n-1}+p^{n}},
$$

то, как показано в [2], система (1) неособым преобразованием $X=M Y$ может быть приведена к виду

$$
\dot{Y}=A_{1} Y+b_{1} \varphi(\sigma), \quad \sigma=c_{1}^{T} Y
$$

где

$$
A_{1}=\left(\begin{array}{ccccc}
0 & 1 & 0 & \ldots & 0 \\
0 & 0 & 1 & \ldots & 0 \\
\ldots & \ldots & \ldots & \ldots & \ldots \\
0 & 0 & 0 & \ldots & 1 \\
-a_{0} & -a_{1} & -a_{2} & \ldots & -a_{n}
\end{array}\right), \quad b_{1}=\left(\begin{array}{c}
0 \\
0 \\
0 \\
\vdots \\
1
\end{array}\right), \quad c_{1}=\left(\begin{array}{c}
-c_{0} \\
-c_{1} \\
-c_{2} \\
\vdots \\
-c_{n-1}
\end{array}\right) .
$$

При этом матрица преобразования $M$ может быть найдена из системы уравнений

$$
A M=A_{1} M, \quad b=M b_{1}, \quad c_{1}=M^{T} c .
$$

Система (12)-(13) является системой каскадного типа. Если система (1) имела аттрактор, то и система (12)-(13), очевидно, также имеет аттрактор, который можно попытаться «клонировать», вводя в систему периодические функции. Тем самым можно попытаться построить экстремально мультистабильную систему, содержащую $(n-1)$-мерную решетку идентичных аттракторов.

Проиллюстрируем реализацию описанной идеи на примере рассмотренной в разделе 2 классической системы Чуа (3)-(5). При выбранных значениях параметров системы ее передаточная функция имеет вид

$$
\chi(p)=\frac{102,13736608+8,50017224 p+8,4562 p^{2}}{-15,037737580544+2,374374715168 p-0,23617016 p^{2}+p^{3}} .
$$

По передаточной функции (15) выпишем систему (12)-(13):

$$
\begin{aligned}
\dot{x}= & y \\
\dot{y}= & z \\
\dot{z}= & 15,037737580544 x-2,374374715186 y+0,23617016 z+ \\
& +\varphi(-102,13736608 x-8,50017224 y-8,4562 z) .
\end{aligned}
$$

Найдем матрицу $M$ линейной замены, решив систему (14):

$$
M=\left(\begin{array}{ccc}
-102,13736608 & -8,50017224 & -8,4562 \\
-0,04397224 & -8,4562 & 0 \\
102,09339384 & 0 & 0
\end{array}\right) .
$$

Численно проинтегрировав систему каскадного типа (16) с начальным условием $Y_{0}=M^{-1} X_{0}=$ $(-0,035909052,-0,167118918,0,197947848)$, находим ее скрытый аттрактор, представленный на рис. 10. 


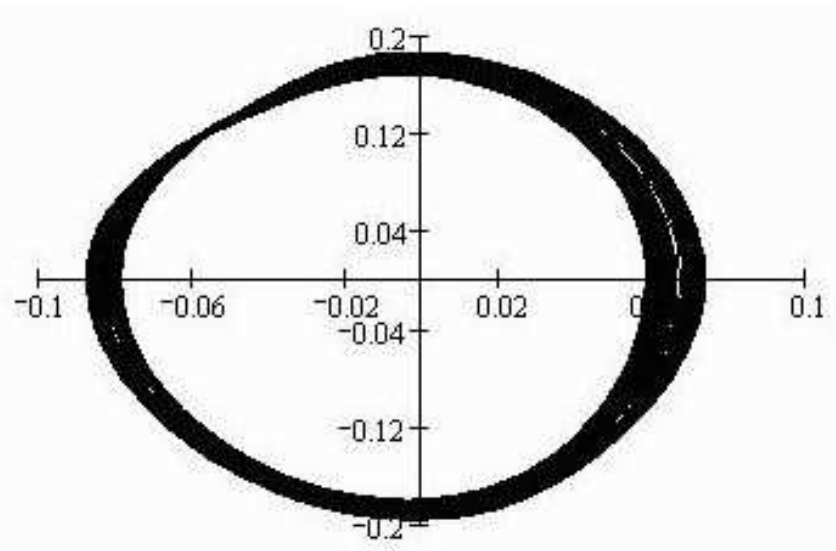

Рис. 10. Скрытый аттрактор системы (16)

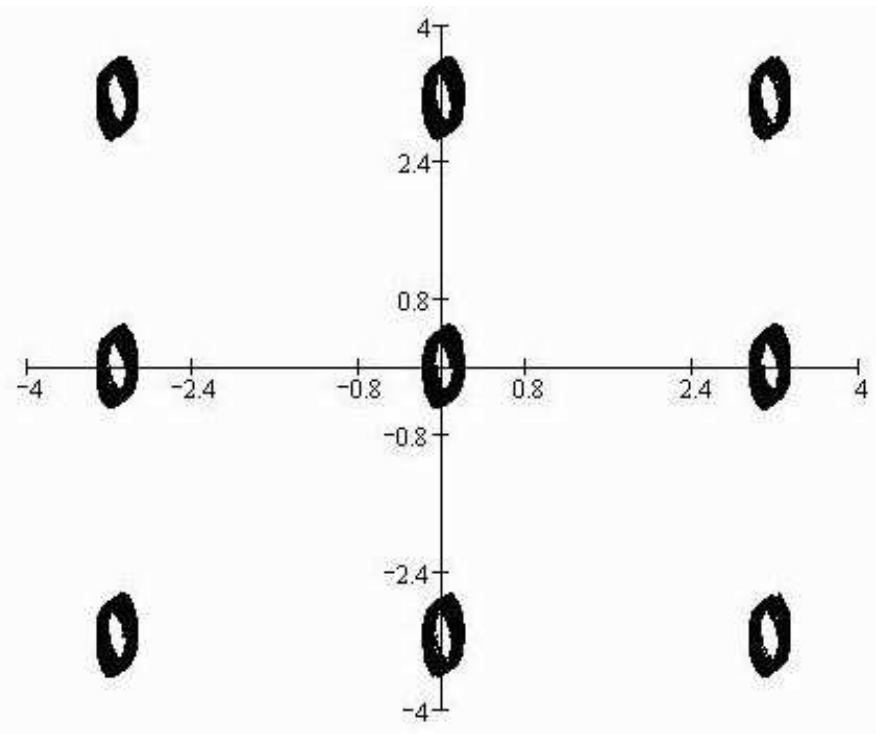

Рис. 11. Двумерная решетка скрытых аттракторов системы (17)

Теперь заменим в системе (16) переменные $y$ и $z$ на $\pi$-периодические функции: $y \rightarrow 0,5 \sin 2 y$, $z \rightarrow 0,5 \sin 2 z$. Полученная после такой замены система

$$
\begin{aligned}
\dot{x}= & 0,5 \sin 2 y, \\
\dot{y}= & 0,5 \sin 2 z, \\
\dot{z}= & 15,037737580544 x-1,1871873575840 \sin 2 y+0,11808508 \sin 2 z+ \\
& +\varphi(-102,13736608 x-4,25008612 \sin 2 y-4,2281 \sin 2 z)
\end{aligned}
$$

содержит двумерную решетку идентичных скрытых аттракторов (см. рис. 11), имеющих одинаковый набор ляпуновских показателей $\Lambda_{1}=0,360, \Lambda_{2}=-0,103, \Lambda_{3}=-2,581$ и размерность Каплана-Йорке $D_{K Y}=2,099$. 
4. Заключение. Метод построения бесконечных решеток странных аттракторов может быть применен в таких инженерных приложениях, как безопасная связь и обнаружение слабых сигналов, когда начальные условия принципиально важны для определения динамики системы. В коммуникациях, базирующихся на использовании хаоса, непредсказуемость начальных условий может дополнительно повысить безопасность связи (см. [21]). Поскольку хаотические системы чувствительны к определенным сигналам и в то же время невосприимчивы к шуму, мультистабильность с бесконечным числом аттракторов обеспечивает возможность перемежающихся переходов между порядком и хаосом, которые полезны для обнаружения слабого сигнала (см. [24]).

В настоящей работе обсуждаются различные подходы к генерированию экстремально мультистабильных систем, содержащих бесконечные решетки скрытых хаотических аттракторов. В частности, показано, что для этой цели может быть использована смещаемая по переменным система каскадного типа, содержащая самовозбуждающийся аттрактор. Особое внимание уделено многомерным моделям систем управления в форме Лурье. Обсуждаются методы преобразования таких систем, позволяющие путем выбора специального базиса приводить их к смещаемым по переменным системам каскадного типа. Таким образом, открывается возможность, генерировать системы с бесконечной решеткой скрытых аттракторов, используя многочисленные примеры существования скрытых аттракторов у систем типа Лурье, не прибегая при этом к исчерпывающему компьютерному поиску.

\section{СПИСОК ЛИТЕРАТУРЫ}

1. Буркин И. М. Скрытые аттракторы некоторых мультистабильных систем с бесконечным числом состояний равновесия// Чебышев. сб. - 2017. - 18, № 4. - С. 127-138.

2. Леонов Г. А. Теория управления. - Изд-во С.-Петерб. ун-та, 2006.

3. Arecchi F. T., Meucci R., Puccioni G., Tredicce J. Experimental evidence of subharmonic bifurcations, multistability, and turbulence in a Q-switched gas laser// Phys. Rev. Lett. — 1982. — 49, № 17. — P. 1217.

4. Bao B., Li Q., Wang N., Xu Q. Multistability in Chua's circuit with two stable node-foci// Chaos. — 2016. - 26(4). - 043111.

5. Burkin I. M., Nguen N. K Analytical-numerical methods of finding hidden oscillations in multidimensional dynamical systems// Diff. Equations. — 2014. - № 50. - P. 1695-1717.

6. Bragin V. O., Vagaitsev V. I., Kuznetsov N. V., Leonov G. A. Algorithms for finding hidden oscillations in nonlinear systems. The Aizerman and Kalman conjectures and Chua's circuits// J. Comput. Syst. Sci. Int. — 2011. — 50, № 4. - P. 511-543.

7. Cicek S., Ferikoglu A., Pehlivan I. A new 3D chaotic system: dynamical analysis, electronic circuit design, active control synchronization and chaotic masking communication application// Optik. — 2016. — № 127 . - P. 4024-4030.

8. Dudkowskia D., Jafari S., Kapitaniak T., Kuznetsov N. V., Leonov G. A. Hidden attractors in dynamical systems// Phys. Rep. — 2016. — № 637. - P. 1-50.

9. Komarov A., Leblond H., Sanchez F. Multistability and hysteresis phenomena in passively mode-locked fiber lasers// Phys. Rev. - 2005. - 71 (5). - 053809.

10. Kuznetsov $N$. V. Hidden attractors in fundamental problems and engineering models. A short survey// Lect. Notes Electr. Eng. - 2016. — № 371. - P. 13-25.

11. Lai Q., Chen $S$. Research on a new 3-D autonomous chaotic system with coexisting attractors// Optik. 2016. - № 127. - P. 3000-3004.

12. Lai Q., Chen S. Generating multiple chaotic attractors from Sprott B system// Int. J. Bifurc. Chaos. 2016. -26 . -21650177

13. Laurent M., Kellershohn N. Multistability: a major means of differentiation and evolution in biological systems// Trends Biochem. Sci. - 1999. - 24 (11). - P. 418-422.

14. Leonov G. A., Burkin I. M., Shepeljavyi A. I. Frequency Methods in Oscillation Theory. - Amsterdam: Kluwer, 1996.

15. Leonov G. A., Kuznetsov N. V, Vagaitsev V. I. Localization of hidden Chua's attractors// Phys. Lett. 2011. — № 375. — P. 2230-2233.

16. Li C., Hu W., Sprott J. C., Wang X. Multistability in symmetric chaotic systems// Eur. Phys. J. Spec. Top. - 2015. - № 224. - P. 1493-1506. 
17. Li C., Sprott J. C. Multistability in the Lorenz system: a broken buttery// Int. J. Bifurc. Chaos. — 2014. $-24(10) .-1450131$.

18. Li C., Sprott J. C., Hu W., Xu Y. Infinite multistability in a self-reproducing chaotic system// Int. J. Bifurc. Chaos. - 2017. - 27 (10). - 1750160.

19. Li C., Sprott J. C., Kapitaniak T., Lu T. Infinite lattice of hyperchaotic strange attractors// Chaos Solitons Fract. - 2018. — № 109. - P. 76-82.

20. Li C., Sprott J. C., Mei Y. An invite 2-D lattice of strange attractors// Nonlin. Dynam. - 2017. — 89, № 4. - P. $2629-2639$.

21. Li Z ., Xu D. A secure communication scheme using projective chaos synchronization// Chaos Solitons Fract. - 2004. - № 22. - P. 477-481.

22. Liu H., Kadi A., Li Y. Audio encryption scheme by confusion and diffusion based on multi-scroll chaotic system and one-time keys// Optik. - 2016. — № 127. — P. 7431-7438.

23. Ujjwal S. R., Punetha N., Ramaswamy R., Agrawal M., Prasad A. Driving-induced multistability in coupled chaotic oscillators: symmetries and riddled basins// Chaos. - 2016. - 26. - 063111.

24. Wang G., Chen D., Lin J., Chen X. The application of chaotic oscillators to weak signal detection// IEEE Trans. Ind. Electron. - 1999. - № 46. - P. 440-444.

25. Xu Q., Lin $Y$., Bao B., Chen $M$. Multiple attractors in a non-ideal active voltage-controlled memristor based Chua's circuit// Chaos Solitons Fract. — 2016. — № 83. — P. 186--200.

26. Ying L., Huang D., Lai Y. C. Multistability, chaos, and random signal generation in semiconductor superlattices// Phys. Rev. E. - 2016. - 93 (6). - 062204.

27. Zeng Z., Huang T., Zheng $W$. Multistability of recurrent neural networks with time-varying delays and the piecewise linear activation function// IEEE Trans. Neural Networks. — 2010. — № 21 (8). — P. 1371—1377.

Буркин Игорь Михайлович

Тульский государственный университет

E-mail: i-burkin@yandex.ru

Кузнецова Оксана Игоревна

Тульский государственный университет 